\title{
Oxidative status for Iraqi young female patients with diabetes mellitus type one
}

\author{
"Perry H. saifullah", "Yahiya D.al-saadi", "Rawaa M. Mohammed Diaa" \\ College of science for women department of chemistry Baghdad university, Treatment and \\ ResearchCenterdiabetesmustansiriya university, Graduate student in college of science for women department \\ of chemistry Baghdad university.
}

\begin{abstract}
:
The study attempts to investigates the relationship between diabetes patients and controls represented by levels of (fasting blood sugar, glutathione_s transferase,blood urea, and Malone di aldehyde)in Iraqi young female patients with DM1 without complications. The study groups consisted of 66 patients with DM1 (15_30years) and 22 controls young female. It was found that the GST was higher in diabetes patients were compared with controls but less over time where( group one slightly higher than group two and more higher than group three) and were found GST not significantly in diabetes patients were compared with controls. In the MDA It was found that the group one and group two of diabetes patients were significantly ( $p$ value $<0.05$ ) between diabetes patients and controls and found MDA less over the time.
\end{abstract}

\section{Introduction}

Diabetes mellitus is a clinically and genetically heterogeneous group of metabolic manifested by high levels of metabolic disorders manifested by abnormally high levels of glucose in the blood, The hyperglycemia is the result of deficiency of insulin secretion caused by pancreatic B-cell resistance to the action of insulin in liver and muscle or combination of these ,frequently this metabolic disarrangement is associated with alteration inadipocyte metabolism, diabetes is syndrome and it is now recognized that chronic hyper glycemia leads to long term damage to different organs including the heart,eyes,kidneys,nervesand vascular system. There are several etiologies for diabetes and although establishing the type of diabetes for each patient is important, understanding the patho physiology of the various forms of the disease is the key appropriate treatment, the current classification of diabetes is based upon the pathophysiology of each form of the disease.

Type one diabetes (B-cell destruction usually leading to absolute insulin deficiency) this form of diabetes, which accounts for only 5_10\% of those with diabetes, previously encompassed by the terms insulin dependent diabetes type one diabetes result from a cellular mediated auto immune destruction of the B-cell of the pancreas, some forms of type one diabetes have no known etiologies. Autoimmune destruction of B-cells has multiple genetic predisposition and is also related to environmental factors that are still poorly define, although patient are rarely obese when they present with this type one diabetes, the presence of obesity is not incompatible with the diagnosis.Increasing evidence in both experimental and clinical studies suggests that oxidative stress plays major role in the pathogenesis of the both types of diabetes Miletus ,free radicals are formed disproportionately in diabetes by glucose oxidation, non enzymatic glycation of proteinsand the simultaneous decline of antioxidant defence mechanisms can lead to damage of cellular organelles and enzymes, increase lipid peroxidation and development of insulin resistance, these consequences oxidative stress can promote the development of complications of diabetes mellitus , change in oxidative stress bio markers, including malondialdehyde,glutathione transferase, blood urea.Glutathione transferase have historically also been called glutathione s_transferase, and it is the latter name that gives rise to the widely used abbreviation G S T, these enzymes catalyze nucleophilic attack by reduced glutathione(GSH) on non polar compounds that contain an electrophilic carbon, nitrogen or sulphar atom. GST is a super family of enzymes that catalyzes the conjucation of xenobiotics and endogenous substances with glutathione, and thereby play a significant role in the inactivation and occasionally the activation of many drugs and xenobiotics, a number of potentially toxic electrophilic xenobiotics (such as acertain carcinogens) are

conjugated to the nucleophilic.

MDA is the most marker that is used to investigate the presence of lipid peroxidation in biological system, the path ways that lead to marker from lipid peroxidation ,MDA is a significant by product during enzymatic synthesis of prostaglandin, it can be removed by renal clearance, it plays a significant role in DNA damage, sister chromatid exchange and carcinogenesis ,MDA is athree carbon molecule with two aldehyde groups which renders it highly reactive with other bio molecules,MDA circulates in plasma either bound to protein or in free form, another protion of MDA is generated in vitro from decomposition of lipid hydroperoxides during sample

preparation. 
Urea is the chief end products of protein metabolism in the body the importance of the urea concentration in the blood lies in its value as an indicator of kidney function, Azotemia(an abnormal increase in plasma urea level) ,they are two types of azotemia the first prerenal azotemia is caused by impaired perfusion of the kidneys due to decreased cardiac output or for any of the former causes, the second post renal azotemia,is caused by an obstruction in the urine out flow such as nephrolithiasis,prostatism, and tumors of the genitourinary tract, the clinical significance of the urea level is usually determined in conjugation with theplasma creatinine level, in prerenal azotemia an increase in the plasma urea level is usually associated with anormal creatinin level where as postrenal azotemia there is an increase inboth the urea and plasma creatinine levels decrease in the urea plasma level may be associated with acute dehydration, malnutrition, pregnancy.

\section{Materials and Methods}

Patient and design. The study group consisted of 66 young female (15-30)years with DM1 divided to three groups $(5,10,15)$ years according to duration of disease and they had no complication , the number of the groups $(24,22,20)$ respectively, this samples collected from the center of diabetes /almustansria university, in January, February 2013.

Meterials.trichloroaceticacide(BDH,England),thio barbutaricacid(H\&w,Germany), deionization distilled water.

Chloro di nitro benzene (H\&W,Germany) phosphate buffer slain (Biomaghreb,sa,france),reduced glutathione(BDH,England), Enzyme reagent ureas $>500 \mathrm{U} / \mathrm{mL}$,bufferd chromagon(phosphate buffer20mmol/1 PH6.9,EDTA2mmol/1,sodium salycilate $60 \mathrm{mmol} / 1$ sodium nitroprusside $3.4 \mathrm{mmol} / \mathrm{l}$ ) alkaline hypochlorite,sodium hypochlorite 10mmol/1,NAOH150mmol/1,Urea standard urea50mg/dl,(spinreact,spain).

Determination of Urea in serum. Urea determined by bring reagents and samples to room temperature, then pipetted in to acuvette $1.0 \mathrm{ml}$ of working reagent in the blank ,sample,standerd Tubes and pippete $10_{\square} 1$ from sample in the sample tube and $10_{\square} 1$ in the standard tube, mixed and incubated for 5 minutes at $37 \mathrm{c}$,then pipetted $1.0 \mathrm{ml}$ from reagent to the blank ,sample, and standard tubes, mix thoroughly and incubated the tubes for 5 minutes at $37 \mathrm{c}$,read the absorbance(A) of the samples and the standard at $600 \mathrm{~nm}$ against the reagent blank.

Determination of Glutathione -s- transferase. Was determined by placed $900_{\mathrm{m}} \mathrm{L}$ of enzyme cocktail $\left(980_{\mathrm{m}} \mathrm{L}\right.$ PBS PH6.5,10 $\mathrm{m}$ of $100 \mathrm{mM}$ CDNB, $10 \mathrm{mM}$ glutathione ) into $1.5 \mathrm{ml}$ quartzes cuvette, incubated at $30 \mathrm{c}$ in spectrophotometer for $5 \mathrm{~min}$, to the blank cuvete added $100 \mathrm{ml}$ PBS and zero spectro, $100 \mathrm{ml}$ were added to sample cuvette and mixed, measured absorbance at $340 \mathrm{~nm}$ for five-minute.

Determination of fast blood Glucose. Was determined by clinically method in the early morning by take drop of blood from patient with diabetes type one were the patient fasting measured the glucose by the diabetes apparatus.

Determination of Malone di aldehyde.Was determined by placed $150_{\square} \mathrm{L}$ of sample(serum), $1 \mathrm{ml}$ of $\operatorname{TCA}(17.5 \%), 1 \mathrm{ml}$ of $\mathrm{TBA}(0.6 \%)$ in the tube and placed $1 \mathrm{ml}$ of $\mathrm{TCA}(17.5 \%), 1 \mathrm{ml} \mathrm{TBA}(0.6 \%)$ in another tube (blank),all tubes mixed by vortex and incubated in100c for 15 minute, and all tubes were cooled ,then placed $1 \mathrm{ml} \mathrm{TCA}(70 \%)$ in the sample tube and $1 \mathrm{ml} \mathrm{TCA}(70 \%)$ in the blank tube mixed and staled for 20 minute at room temperature, then centrifuged $4000 \mathrm{rpm}$ for 15 minute and the supernatant was taken out for mesearing at $532 \mathrm{~nm}$.

\section{Results and Discussion}

Glutathione s-transferase constitute a major protective mechanism against oxidative stress low levels of GSH in diabetes patients are found to potentiate the effects of the increased reactive oxygen species, increased of GST activity in diabetes patients independent of their glycemic status there is no significant difference between diabetics and non diabetics.

Increase total GST levels may be due to a compensatory mechanism of anti oxidant to combat the oxidative stress in diabetic conditions with or without complication. The regulation of GST

subjected to a complex set of endogenous and exogenous parameters, as well as large number of xenobiotics inducing agents. 
The chronicity of the disease along with treatment modalities might have played a significant role in the subjects included in diabetic group were enrolled without considering their glycemic control status.

Urea in the serum was found not significantly in three groups compared with control groups that mean no defect in kidney functions because all patients groups had diabetic without complications.

The increase of MDA levels in group one and group two reflected to an increase in lipid peroxidation initiatedby ROSs ,so that imbalance between anti oxidant production and removal of ROSs lead to oxidative stress, but in group three MDA levels decreased and were found not significantly compared with controls because different reasons: no system in the life style, lack of sport, no right nutrition ,long time of insulin doses, commitment of periodic examination, genetics, shocks, and another diseases.

The study attempts to investigates the relationship between diabetes patients and controls represented by levels of (fasting blood sugar, glutathione_s transferase ,blood urea, and Malone di aldehyde)in Iraqi young female patients with DM1 without complications. The study groups consisted of 66 patients with DM1 (15_30years) and 22 controls young female. It was found that the GST was higher in diabetes patients were compared with controls but less over time where( group one slightly higher than group two and more higher than group three) and were found GST not significantly in diabetes patients were compared with controls. In the MDA It was found that the group one and group two of diabetes patients were significantly ( $p$ value $<0.05$ ) between diabetes patients and controls and found MDA less over the time.

Table 1: GST activity in all patient groups and control group

\begin{tabular}{|c|c|c|c|c|}
\hline Groups & Controls & Group1 & Group2 & Group3 \\
\hline $\mathrm{N}$ & 22 & 24 & 22 & 20 \\
\hline Mean & 216.6127 & 255.5188 & 254.5486 & 93.4253 \\
\hline Std. Dev. & 65.5468 & 98.7162 & 72.9880 & 21.0047 \\
\hline Std. Error & 13.9746 & 20.1503 & 15.5611 & 0.699 \\
\hline probability & & 0.028 & 0.725 & \\
\hline
\end{tabular}

The results are presented as GST activity in group one significantly but in group two and three not found any . (significantly relation between diabetic patients and control groups where $\mathrm{p}$ value $(<0.05$

Table 2: MDA concentration in all patient groups and control group

\begin{tabular}{|c|c|c|c|c|}
\hline Groups & Controls & Group1 & Group2 & Group3 \\
\hline $\mathrm{N}$ & 22 & 24 & 22 & 20 \\
\hline Mean & 1.2020 & 3.3573 & 2.6356 & 1.3323 \\
\hline Std. Dev. & 1.1752 & 3.2152 & 2.0889 & 1.1932 \\
\hline Std. Error & 0.2932 & 0.7175 & 0.6305 & 0.4457 \\
\hline probability & & 0.001 & 0.0001 & 0.147 \\
\hline
\end{tabular}

The results are presented as MDA concentration in group one and group two are significantly but in group three not found any significant relation between patient and controls where $\mathrm{p}$ value is $(<0.05)$

Table 3: urea in serum concentration in all patient groups and control group

\begin{tabular}{|l|l|l|l|l|}
\hline Groups & Controls & Group1 & Group2 & Group3 \\
\hline N & 22 & 24 & 22 & 20 \\
\hline Mean & 23.5455 & 25.3750 & 25.5909 & 23.6500 \\
\hline Std. Dev. & 3.3198 & 4.6794 & 4.9728 & 3.5433 \\
\hline Std. Error & 0.7078 & 0.9551 & 1.0602 & 0.7923 \\
\hline probability & & 0.145 & 0.111 & 0.936 \\
\hline
\end{tabular}

The results are presented as urea in serum in group one ,two and three not found any significant relation between patient groups and control group. $\mathrm{P}$ value $(<0.05)$. 
Table 4: fast blood glucose concentration between all patient groups and control group

\begin{tabular}{|c|c|c|c|c|}
\hline Groups & Controls & Group1 & Group2 & Group3 \\
\hline N & 22 & 24 & 22 & 20 \\
\hline Mean & 103.4091 & 265.05417 & 242.0455 & 243.9500 \\
\hline Std. Dev. & 3.2755 & 87.77490 & 109.0777 & 85.6292 \\
\hline Std. Error & 0.6983 & 17.91698 & 23.2554 & 19.1472 \\
\hline probability & & 0.0001 & 0.0001 & 0.0001 \\
\hline
\end{tabular}

The results presented as fast blood sugar in group one, two and three we are found group one ,two and group three are significantly relation between diabetic patient and control $\mathrm{p}$ value $(<0.05)$.

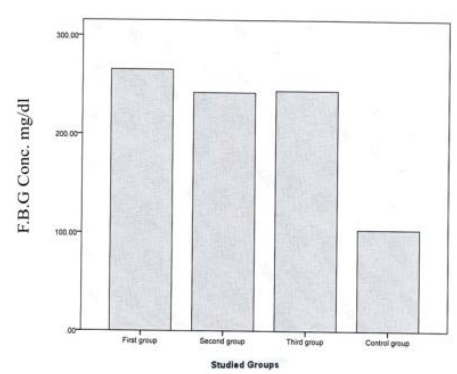

Fig.1: fasting blood glucose concentration between diabetic patients and control

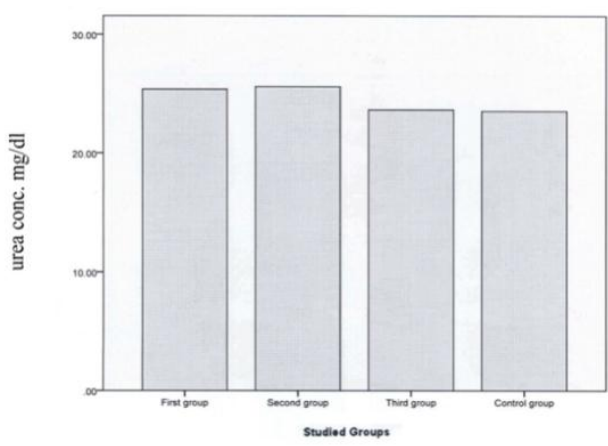

Fig. 2: urea concentration in serum between diabetic patients and control

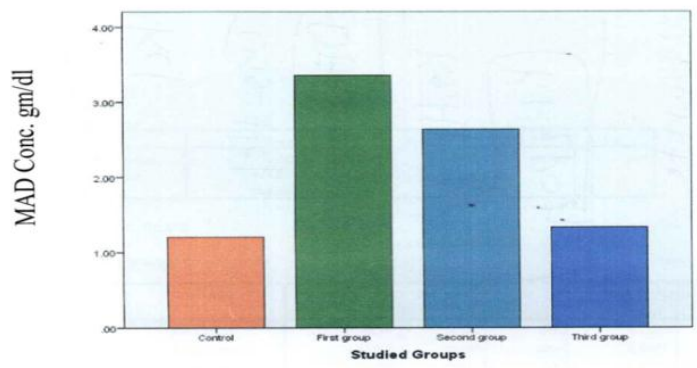

Fig. 3: MDA concentration between diabetic patients and control.

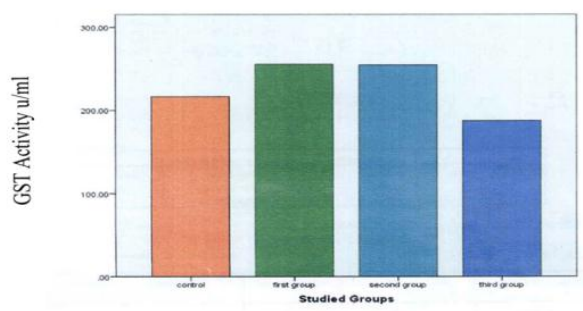

Fig .4 : GST activity between diabetic patients and control 
[1] Diabetes care , volume 35, supplement1, January 2012.

[2] periodontology medical, volume 44, 2007, 127-153.

[3] International Journal of research inAuruvede and pharmacy , volume1, Issue1,sep _oct 2010,33-42.

[4] Al- Kazzaz,F.F.; Saif-Alah,P.H ; Nasif,Z.N(2005) Molecular Characterization and Determination the Level of Oxidation Stress and Some Anti Oxidant in Iraqi Women With Breast Tumors ,Msc.thesis , college of science , Al-Mustansiriyah University.

[5] Guengerich FP, Their R, persmark M., Taylor T.B., pemble S.E., Ketterer B.: conjucation of carcinogens by theta glasss glutathione stransferase :Mechanisms and relerance to varicetion in human risk. Pharmacogenetics (1995);5:3.

[6] Guengerich F.P. : metabolism and genetoxicity of dihabalkanes .Adv. pharmacol.(1994); 27:211.

[7] Tsuchida s; sato K:Glutathione s-transefersase and cancer CRC.Crit.Rev.Biochem.Mol.(1992) 27:337.

[8] Tew K.D: Glutathione -associated enzymes in anticancer drug resistance. Cancer Res.(1996); 54:4313.

[9] Jarrell B.E, and carbans R.A : National Medical services for independent study .(surgery $3^{\text {rd }}$ ed. ) Williams\& wilkers Co/ Baltimore(1996); chap.23.

[10] Dhalla N.S; Temsah R.M, Netticudan T: the role of oxidative stress in cardiovascular disease J. Hypertens.(2000); 18:655-673.

[11] Mannervik,B. the isoenzyme of glutathione transferase . Adv. Enzymol.Relat.Areas Mol.Biol.57,357-417(1985).

[12] boyland,E. and chasseaud,L.F. the role of Glutathione s transferase in Mercaptic Acid Biosynthesis . Adv.Enzymol.32 173-219(1969).

[13] chaney ,AL,and E.P,clin.chem.8:132(1962).

[14] searcy,R.L, Reardon,J.E, and Foreman,J.a.AM.j.clin. Technol.33:15_20(1967).

[15] Young Ds.Effects of drugs on clinical laboratory tests, $5^{\text {th }}$ ed. AAcc press, 2000

[16] patton ,c.s,m and crouch, s.R.Anal.chem.49: 464(1977).

[17] tiatz N.W, clinical Guideto Laboratory test, $3^{\text {rd }}$ Edition W.B.saunders co.philadelphia,PA.(1995).

[18] friedman and Young.Effects of disease on clinical laboratory tests. $5^{\text {th }}$ ed.AACC(press2000).

[19] Frankel E.N.; Recent advances in lipid peroxidation .J.sci. food Agric.(1991; 54 : 495_ 511.

[20] Handelman G.J.;Evalution of oxidative stress in dialysis patients. Blood purif . (2000) ; 18: 343-349.

[21] Liu J.Yeo H.C, Doniger S .J , Ame B.N: Assay of aldehydes from lipid peroxidation : gas chromatograraphy mass spectrometry compard to thio barbituric acid . Anal Biochem.(1997); 945:161-166.

[22] Yeo H.C.HelbackH.J, chya D.W. Ames B.N; Assay of malone di aldehyde in biological fluid by gas chromatography -mass spectrometry.Anal.biochem.(1994);220:391-396.

[23]OhkawaH;ohishi N.I Yagi K Assay for lipid peroxides in animal tissue by thiobarbituric acid reaction.Anal. Biochemist(1979),75-78. [24]GerristenW.B;A artsL.P.;MorshuisW.J,Hass F.J:Indiees of oxidative stress in urin of patients undergoing coronary artery by pass grafting. Eur.JA.clin.chem.clin.Biochem.(1997);35:737-742.

[25] Baraczyl-Kuoma,M; Gutowicz, M, Kaomierczak, B. and Sawicki, J. 2004. Gluthione s- transferase as a target for tri cyclic antidepressants in human brain.Acta Biochemica polonica.51(1):pp. 207-212. 